\title{
Correction to: Lifespan-extending property of 6-shogaol from Zingiber officinale Roscoe in Caenorhabditis elegans
}

Eun Byeol Lee ${ }^{1,2} \cdot$ Jun Hyeong Kim ${ }^{1}$ Yeong Jee Kim ${ }^{1}$ Yun Jeong Noh ${ }^{1}$. Su Jin Kim ${ }^{1}$ - In Hyun Hwang ${ }^{1}$ Dae Keun Kim ${ }^{1}$

Published online: 6 February 2019

(C) The Pharmaceutical Society of Korea 2019

Correction to: Arch. Pharm. Res. (2018) 41:743-752 https://doi.org/10.1007/s12272-018-1052-0

The author would like to include conflict of interest statement of the online published article. The correct conflict of interest statement should read as:
Conflict of interest The authors declare no conflict of interest.

The original article can be found online at https:// doi.org/10.1007/s12272-018-1052-0.

Dae Keun Kim

dkkim@woosuk.ac.kr; dkkim1574@naver.com

1 College of Pharmacy, Woosuk University, Jeonju 55338, Republic of Korea

2 Department of Agro-food Resources, Rural Development Administration, Wanju 55365, Republic of Korea 